\title{
Factors associated with health status and psychological distress among cardiac arrest survivors treated with an implantable cardioverter-defibrillator
}

Johan Israelsson, Department of Cardiology, Kalmar County Council, Kalmar, Sweden, johani@ltkalmar.se

Ingela Thylén, Department of Medical and Health Sciences, Division of Nursing Science, Linköping University, Linköping, Sweden Anna Strömberg, Department of Medical and Health Sciences, Division of Nursing Science, Linköping University, Linköping, Sweden

Anders Bremer, Faculty of Health and Life Sciences, Linnaeus University, Växjö, Sweden

ICARE

Kristofer Årestedt, Faculty of Health and Life Sciences, Linnaeus University, Kalmar, Sweden

\section{Purpose}

To explore factors associated with health status and psychological distress among cardiac arrest (CA) survivors treated with an implantable cardioverter-defibrillator (ICD), and to compare their health status with a general Swedish population

\section{Material and methods}

- Cross-sectional study including 990 CA survivors treated with an ICD

- Linear regression analyses were used to explore factors associated with EQ index, EQ VAS (health status), HADS anxiety and HADS depression (psychological distress)

\section{Results}

The following factors were independently associated with worse outcome in all four final regression models:

Being unemployed

Suffering more comorbidity

Perceiving less control Having a type D personality
Health status among ICD-treated cardiac arrest survivors compared to a Swedish general population*

$\begin{array}{llllll}\text { Variable, } m \text { (SD) } & \begin{array}{l}\text { All, } \\ n=1990\end{array} & \begin{array}{l}\text { CA survivors, } \\ n=990\end{array} & \begin{array}{l}\text { General } \\ \text { population, } \\ n=1000\end{array} & \begin{array}{l}p \text {-value } \\ \text { E }\end{array} & \begin{array}{l}\text { Eta square } \\ \left(n^{2}\right)^{\mathrm{b}}\end{array} \\ \text { EQ index } & 0.81(0.21) & 0.84(0.20) & 0.79(0.21) & <0.001 & 0.022 \\ \text { EQ mobility } & 1.24(0.43) & 1.27(0.45) & 1.21(0.41) & 0.002 & 0.005 \\ \text { EQ self-care } & 1.04(0.23) & 1.05(0.23) & 1.04(0.23) & 0.278 & 0.000 \\ \text { EQ usual activities } & 1.16(0.42) & 1.20(0.46) & 1.12(0.37) & <0.001 & 0.014 \\ \text { EQ pain/discomfort } & 1.55(0.56) & 1.40(0.53) & 1.70(0.55) & <0.001 & 0.073 \\ \text { EQ anxiety/depression } & 1.29(0.49) & 1.27(0.47) & 1.30(0.50) & 0.382 & 0.000 \\ \text { a Mann-Whitney U test } & & & & & \\ \text { * Matched for age and gender on a group level } & \\ \text { b Effect size measured by Eta square (small=0.01-0.06, medium }=0.06-0.14 \text {, large >0.14) }\end{array}$

\section{Conclusions}

- Health status among most ICD treated CA survivors is good

- Several factors predicting worse health status and more psychological distress were identified

- These results might be used when screening patients for health problems during post CA follow-up and when developing health promoting interventions 